\title{
Versatile Video Coding for 3.0 Next Generation Digital TV in Brazil
}

\author{
Thibaud Biatek \\ Mohsen Abdoli \\ Mickael Raulet \\ Adam Wieckowski \\ Christian Lehman \\ Benjamin Bross \\ Philippe De Lagrange \\ Edouard François \\ Ralf Schaefer \\ Jean Lefeuvre
}

CITE THIS ARTICLE

Biatek,Thibaud; Abdoli,Mohsen; Raulet,Mickael; Wieckowski, Adam; Lehman, Christian; Bross, Benjamin; De Lagrange, Philippe; François, Edouard; Schaefer, Ralf and Lefeuvre, Jean; 2021. Versatile Video Coding for 3.0 Next Generation Digital TV in Brazil . SET INTERNATIONAL JOURNAL OF BROADCAST ENGINEERING. ISSN Print: 2446-9246 ISSN Online: 2446-9432. doi: 10.18580/setijbe.2021.1. Web Link: http://dx.doi.org/10.18580/setijbe.2021.1 


\title{
Versatile Video Coding for 3.0 Next Generation Digital TV in Brazil
}

\author{
Thibaud Biatek ${ }^{1}$, Mohsen Abdoli ${ }^{1}$, Mickael Raulet ${ }^{1}$, Adam Wieckowski ${ }^{2}$, Christian Lehman ${ }^{2}$, \\ Benjamin Bross ${ }^{2}$, Philippe De Lagrange ${ }^{3}$, Edouard François ${ }^{3}$, Ralf Schaefer ${ }^{3}$ and Jean Lefeuvre ${ }^{4}$
}

${ }^{1}$ ATEME, ${ }^{2}$ Fraunhofer HHI, ${ }^{3}$ InterDigital, ${ }^{4}$ Télécom Paris

\begin{abstract}
In the past few decades, the video broadcast ecosystem has gone through major changes; Originally transmitted using analog signals, it has been more and more transitioned toward digital, leveraging compression technologies and transport protocols, principally developed by MPEG. Along this way, the introduction of new video formats was achieved with standardization of new compression technologies for their better bandwidth preservation. Notably, SD with MPEG-2, HD with H.264, 4K/UHD with HEVC. In Brazil, the successive generations of digital broadcasting systems were developed by the SBTVD Forum, from TV-1.0 to TV-3.0 nowadays. The ambition of TV-3.0 is significantly higher than that of previous generations as it targets the delivery of IPbased signals for applications, such as $8 \mathrm{~K}$, HDR, virtual and augmented reality. To deliver such services, compressed video signals shall fit into a limited bandwidth, requiring even more advanced compression technologies. The Versatile Video Coding standard (H.266/VVC), has been finalized by the JVET committee in 2021 and is a relevant candidate to address the TV3.0 requirements. VVC is versatile by nature thanks to its dedicated tools for efficient compression of various formats, from $8 \mathrm{~K}$ to $360^{\circ}$, and provides around $50 \%$ of bitrate saving compared to its predecessor HEVC. This paper presents the VVC-based compression system that has been proposed to the SBTVD call for proposals for TV-3.0. A technical description of VVC and an evaluation of its coding performance is provided. In addition, an end-to-end live transmission chain is demonstrated, supporting $4 \mathrm{~K}$ real-time encoding and decoding with a low glass-to-glass latency.
\end{abstract}

Index Terms - DTT, OTT, VVC, 8K, 4K, HDR, HFR, Broadcast

\section{INTRODUCTION}

$\mathrm{T}$ HE SBTVD Forum (Sistema Brasileiro de TV Digital [1]) is a Brazilian organization responsible for development of digital television in Brazil. The organization gathers more than eighty members, private and public, covering the complete ecosystem, including broadcasters, manufacturers, institutions, and universities. Over the years, SBTVD enabled the transition from analog to digital, releasing a number of specifications issued by the Brazilian Association of Technical Standards (ABNT). In its most recent version [2], the coding technology used remains H.264/AVC [3] even though advanced picture format such as High Dynamic Range (HDR) is covered.

In July 2020, SBTVD issued a call for proposals aiming at extending the current digital television system to new usecases and futuristic applications. Among these applications, video format such as $8 \mathrm{~K}, \mathrm{HDR}, \mathrm{AR} / \mathrm{VR}$ shall be supported and delivered over broadcast, or hybrid broadband/broadcast networks, based on IP-centric protocols. To enable the delivery of such signals with high Quality of Experience (QoE) for the viewer, the usage of advanced compression systems is required.

Versatile Video Coding (VVC) [4][5], also known as H.266, is the most recent video coding standard issued by the Joint Video Experts Team (JVET) of ITU-T and ISO/IEC and approved in July 2020. VVC is the successor of High Efficiency Video Coding (HEVC) standard [6], providing around $50 \%$ of bitrate saving for the same visual quality. VVC has been designed to address a wide range of applications (e.g. broadcast, streaming, layered coding) and formats (e.g. 4K/8K, HDR, VR-360, Screen-Content), and is then a relevant candidate to address TV-3.0 requirements.

VVC was proposed by a consortium formed by ATEME, DiBEG, Fraunhofer $\mathrm{HHI}$ and InterDigital as coding technology for TV3.0 in November $30^{\text {th }} 2020$ and since then is going through the evaluation procedures. Beside VVC, other codecs have been proposed, such as HEVC, LCEVC [7] and AVS3 [8]. Together with the formal submission, an endto-end delivery scheme was provided enabling to demonstrate the VVC readiness and use-cases in a live environment. The demonstrator includes a live VVC headend, an origin-/adserver, and a video player capable of live VVC decoding and ad-insertion. In this paper, the VVC proposal brought to SBTVD for TV3.0 is thoroughly described, including technology, performance and demonstrator.

The rest of the paper is organized as follows. First, Section II introduces the TV-3.0 project, highlighting the required key features, evaluation procedure and timeline. Second, the VVC compression technology is presented in Section 0. The Section IV presents the end-to-end transmission chain delivered to SBTVD to assess the relevance of VVC in a live environment. Finally, the paper is concluded in Section V.

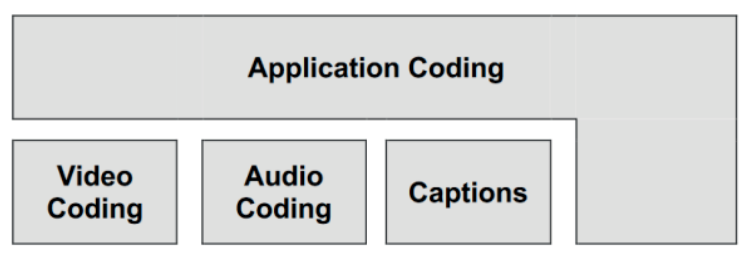

Transport Layer 
Fig. 1: TV-3.0 Architecture [9]

\section{THE TV-3.0 PROJECT}

\section{A. Overview}

The TV3.0 call for proposals has been issued in July 2020 by the SBTVD forum [9], in order to proceed beyond the TV2.5 project, enabling enhanced features and new applications. The TV-3.0 architecture is composed of a number of components, described in Fig. 1, covering the application coding, audio/video coding, captions, transport and physical layers. It is expected that the system will rely on IP protocols for both over-the-air or over-the-top delivery. In addition, the system shall support accessibility and public-safety features which are included as requirements. In this paper, the response that proposes VVC to this call as the video-coding component is presented.

\section{B. Video Coding Requirements}

The TV3.0 video coding component is expected to provide state-of-the-art compression efficiency for live services with a minimal glass-to-glass latency. The native support of HDR and UHDTV is a requirement for the broadcast/broadband delivery, while the support for $8 \mathrm{~K}$ resolution is only restricted to broadband. It is desired that a second video stream can be encoded and delivered for sign language purpose. In addition, support for public safety applications such as emergency warning or sign-language video is desired, as well as an anticipation of immersive technologies support (i.e VR, AR, $\mathrm{XR}, 3 \mathrm{DoF}$ and $6 \mathrm{DoF}$ ). From an application perspective, the selected technology shall support seamless and frameaccurate stream splicing or ad-insertion for broadcast, broadband or hybrid delivery modes. In order to address hybrid use-cases, it is also expected that the selected codec supports scalable and layered coding. In order to be addressed by the proponents, SBTVD split all these features in several use-cases and requirements, reported in the Table 1.

Table 1 : TV-3.0 Video Coding Use-cases [9]

\begin{tabular}{|c|l|}
\hline ID & Description \\
\hline VC1 & $\begin{array}{l}\text { Provide improved video resolution, adequate } \\
\text { to consumer electronics display evolution. }\end{array}$ \\
\hline VC2 & $\begin{array}{l}\text { Provide improved video dynamic range and } \\
\text { color space, adequate to consumer electronics } \\
\text { display evolution. }\end{array}$ \\
\hline VC3 & $\begin{array}{l}\text { Provide sharp images (reducing motion blur), } \\
\text { even on content with fast motion (e.g. sports, } \\
\text { action movies). }\end{array}$ \\
\hline VC4 & $\begin{array}{l}\text { Provide state-of-the-art coding efficiency, to } \\
\text { allow better quality video in limited capacity } \\
\text { channels (over-the-air or the Internet). }\end{array}$ \\
\hline VC6 & $\begin{array}{l}\text { Provide live video with minimum end-to-end } \\
\text { latency. }\end{array}$ \\
$\begin{array}{l}\text { Enable second video stream with a sign } \\
\text { language interpreter to be optionally activated } \\
\text { by the user (to be rendered at the side of the } \\
\text { main video, that should be proportionally } \\
\text { downscaled to fit the horizontal space left, } \\
\text { with no overlap; an optional background still } \\
\text { image can be defined by the broadcaster). }\end{array}$ \\
\hline VC7 & $\begin{array}{l}\text { Enable emergency warning information } \\
\text { delivery using sign language video. }\end{array}$ \\
\hline VC8 & Enable new immersive video services. \\
\hline
\end{tabular}

\begin{tabular}{|c|l|}
\hline VC9 & $\begin{array}{l}\text { Enable seamless decoding and A/V } \\
\text { alignment. }\end{array}$ \\
\hline VC10 & $\begin{array}{l}\text { Enable interoperability with different } \\
\text { distribution platforms (e.g. DTT, cable, IPTV, } \\
\text { DTH satellite, fixed broadband, 4G/5G } \\
\text { mobile broadband, home network). }\end{array}$ \\
\hline VC11 & $\begin{array}{l}\text { Enable scalability (e.g. to improve over-the- } \\
\text { air video quality with an Internet-delivered } \\
\text { enhancement layer) and extensibility (support } \\
\text { new settings and/or features in the future, in a } \\
\text { backward-compatible way). }\end{array}$ \\
\hline
\end{tabular}

In the TV-3.0 CfP response jointly submitted by ATEME, Fraunhofer HHI and InterDigital, VVC has been proposed as technology addressing the complete range of mandatory usecases defined by SBTVD. DiBEG also responded to the CfP with $\mathrm{VVC}$ and joint during the evaluation process the proposal of ATEME, Fraunhofer HHI and InterDigital. The video quality aspects $\mathrm{VC} 1$ to $\mathrm{VC} 4$ are fulfilled thanks to the approach used by JVET to design VVC which is tailored to efficiently address all formats (SD to $8 \mathrm{~K}$ ), dynamic range (SDR to HDR) and type of content (e.g. gaming, sport, movie, screen content, video conferencing) while achieving a maximal compression efficiency. The capability of delivering VVC compression in a live workflow (VC5) is addressed, demonstrated by VVC support in ATEME live encoding platform already demonstrated on the field [10][11].

The low-latency delivery case VC6 can also be addressed by VVC when combined with CMAF Low-Latency and HTTP Chunk Transfer Encoding [12]. VVC is fulfilling the application-oriented use-cases VC6 and VC7 by encoding the second video streams in an efficient manner. VVC natively supports VR-360 coding through specific high-level mechanisms and is thus addressing VC8. In addition, VVC can also be used as core 2D video codec in a V-PCC [13] compression engine and thus can be used to compress volumetric data for $\mathrm{AR} / \mathrm{VR}, 3 \mathrm{DoF}$ or $6 \mathrm{DoF}$ applications. VVC through its defined picture types and encapsulation in ISOBMFF supports seamless splicing or ad-insertion (VC9). The interoperability of VVC with different distribution platforms (VC10) was demonstrated during several on-field trials [10][11]. Finally, VVC natively supports scalable coding (VC11).

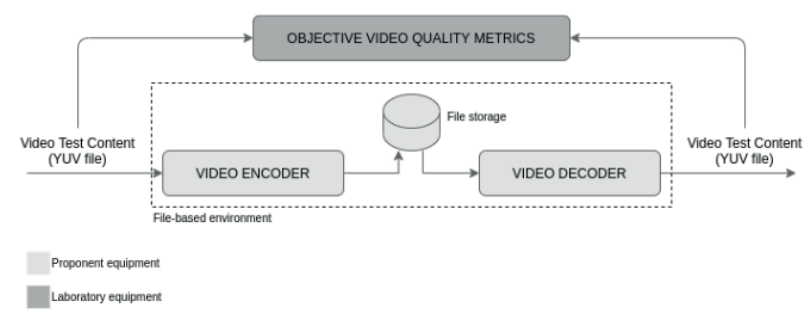

Fig. 2 : Evaluation procedure for non-real-time tests [14]
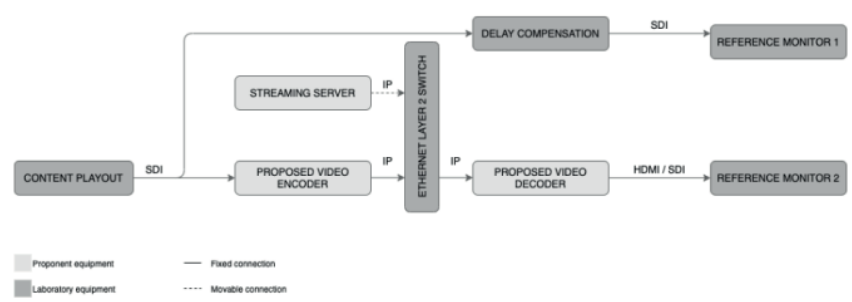

Fig. 3 : Evaluation procedure for real-time tests [14] 


\section{Process and timeline}

It is specified that TV-3.0 is organized in two phases, with dedicated timelines. The first phase consisted of a formal submission, including contact information and how the proposed technology addresses the use-cases' requirements. The first phase response due date was expected on November $30^{\text {th }} 2020$. The second phase, expected to be delivered on January $29^{\text {th }} 2021$, comprised full specification of the solution as well as resources required to evaluate and test the proponents.

In order to evaluate and test the proposed technologies, the proponents should deliver equipment, following architectures described in Fig. 2 and Fig. 3, for non-real-time and real-time tests respectively. This includes video encoder, video decoder, streaming server and file storage. In terms of timeline, the evaluation takes place from July 2021 to December 2021.

\section{Versatile VIDEO CODING}

\section{A. Overview}

Significantly higher performance and more versatility compared to HEVC have been two main elements in the VVC standardization agenda. To meet these requirements, several tools and functionalities were integrated into $\mathrm{VVC}$ in the course of its standardization.

From the versatility point of view, VVC aims to address compression of applications such as $8 \mathrm{~K}$, screen content, immersive video, and multi-resolution over-the-top streaming. At the same time, higher compression efficiency was achieved by introducing advanced coding tools to exploit signal redundancies that are either specific to abovementioned applications, or simply are more efficient than in the previous standards (e.g. HEVC).

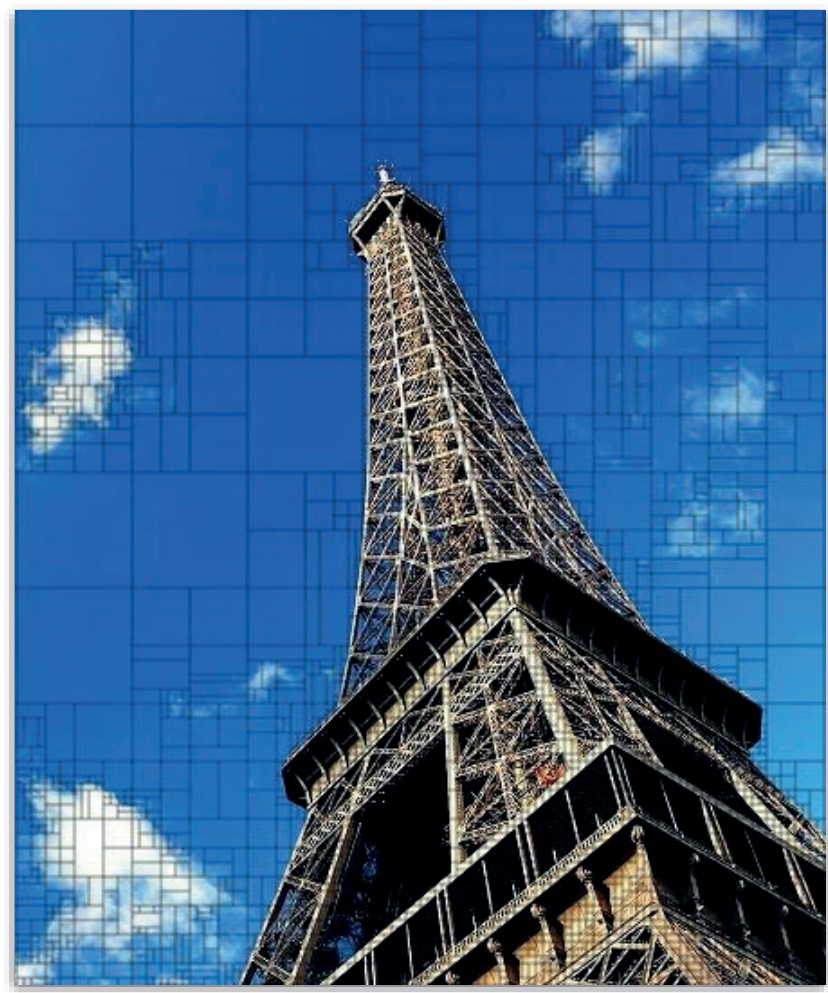

Fig. 4 : Illustration of VVC partitioning

\section{B. Core Coding Tools}

New tools adopted in VVC can roughly be categorized into four groups: partitioning, intra/inter prediction, residual coding, and in-loop filtering.

\section{1) Partitioning}

By far, the main advantage of VVC compared to other video codecs in terms of core coding tools, is its incredibly flexible partitioning scheme [15]. In addition to larger Coding Unit Tree (CTU) size (up to 128 compared to 64, in HEVC), this scheme allows splitting different regions of image in a way that their local is more appropriately represented for spatial and temporal prediction. Compared to HEVC's Quad-Tree (QT), this algorithm of VVC is integrated with binary and ternary (horizontal and vertical) splits in addition to quad split. Consequently, this partitioning method of VVC is known as Multi-Type Tree (MTT). Erro! Fonte de referência não encontrada. shows an example of how flexibly the MTT partitioning of VVC can split regions of an image based on its content complexity.

\section{2) Prediction}

Tens of new coding modes have improved both intra [16] and inter [17][18] prediction performance. In intra prediction, the main contributions are the use of 67 Intra Prediction Modes (IPM) instead of 35 (as in HEVC), Position-Dependent Prediction Combination (PDPC), data-driven block prediction called Matrix-based Intra Prediction (MIP), the possibility of explicitly choosing the reference line, Intra SubPartitioning (ISP) for short-distance pixel prediction, and last, but not least, advanced chroma intra prediction tools such as Cross-Component Linear Model (CCLM).

3) Residual coding

VVC introduces several new ways of encoding prediction residual. This results in a significant overall compression efficiency gain on different types of content [19]. Firstly, VVC allows rectangular transforms for encoding prediction residual in the MTT partitioning. This aspect helps in significant rate saving that was used in HEVC to split rectangular residual blocks into square shapes and transmit separately. Furthermore, VVC is integrated with more transform kernels using discrete sine and cosine transforms (DST, DCT), precisely, DST-I, DCT-II, DCT-V, DST-VII, DCT-VIII, compared to only DCT II, DST VII (limitedly) in HEVC. In addition to traditional separate transformation, VVC also allows a new mode called Low-Frequency NonSeparable Transforms (LFNST), that performs a low complexity non-separable transformation on the residual. This aspect alone accounts for about 2\%-3\% additional compression efficiency gain compared to HEVC.

As screen content coding has usually very specific signal characteristics, VVC is equipped with a dedicated residual coding method, called Transform Skip (TS). As the name suggests, TS performs the quantization step in the pixel domain without any transformation in between [19]. Finally, Dependent Quantization (DQ) enables a switching between two quantizers for decoding each transform coefficient. This choice depends on the previous quantized coefficient's value and a pre-defined state-machine.

4) In-loop filtering

As the processing capacity of both encoder and decoder sides have been significantly improved compared to the last decade, VVC has integrated several in-loop processing and filtering technologies that were not affordable in previous standards [20]. Adaptive Loop Filter (ALF) is a new 
technique, which includes filter shapes, precision, and adaptive clipping processes, in order to tune the filtering parameters both in the sub-blocks and Coding Tree Blocks (CTB) levels. ALF is adaptive in the sense that the filtering coefficients are signaled in the bitstream and can be designed based on image content and distortion of the reconstructed picture. Moreover, a variation of the ALF, called CrossComponent ALF has also been adopted in VVC, which uses the luma sample values to refine the chroma sample values within the ALF calculation. Finally, Luma Mapping with Chroma Scaling (LMCS) is another pre-processing and inloop technique to exploit the dynamic range of the signal. Precisely, LMCS consists of two steps of the Luma Mapping (LM), which remaps the luma code values, and the Chroma Scaling (CS), which allows flexible adjustment between luma and chroma adjustment.

\section{High-Level Features}

VVC has introduced new high-level syntax features [21]. Through its HLS, VVC offers flexible control over tools and features that deal with so-called versatile applications (e.g., layered coding, 360, virtual reality, screen content etc). Examples of such HLS functionalities are as follows. The concept of sup-pictures, tied with the newly introduced Picture Header (PH) and Decodable Capability Information (DCI), flexibly allows random access to subsets of the bitstream, representing localized regions of interest. Thanks to these dedicated syntaxes, subpictures can be arbitrarily recomposed without header rewriting.

Reference Picture Resampling (RPR) is another functionality that allows layered coding and is controlled in the SPS. Other than the traditional use-cases of scalability, RPR potentially saves significant coding efficiency by enabling open GOP in adaptive streaming applications, where the resolution changes frequently. Last, but not least, the support of Screen content coding (SCC) has been carefully considered in the VVC standardization, by dedicating several coding tools that are switchable in different levels of the HLS, in order to limit their usage where their particular content is present [22].

\section{Performance}

The initial agenda of VVC in terms of performance vs. complexity tradeoff was to strictly limit the decoder side complexity, while allowing a certain level of complexity increase at the encoder side. Consequently, software-based decoding of VVC streams (using all tools) is less than twice as complex as decoding of HEVC streams. Given the 10-year gap between the release of the standards, this complexity increase has been considered acceptable by decoder manufacturers since it can be afforded by their state-of-theart technologies.

The VVC encodings for objective and subjective evaluation have been performed using the Fraunhofer Versatile Video Encoder, VVenC, a software VVC encoder implementation, in version 1.0.0 [23]. It is freely available under a 3-clause BDD license [24]. While providing very good compression efficiency, it is only suitable for offline usage. The objective performance evaluation results for three tests scenarios [14] are reported and compared to the HEVC test model (HM) encoder anchor:

- Test 1 contains two test cases requiring coding of video having different spatial resolutions from 720p to 4320p, one (TC 1.1) for Hybrid Log Gamma (HLG) High
Dynamic Range (HDR) video and one for Perceptual Quantizer (PQ) HDR video (TC 1.2).

- Test 3 includes one case (TC 6.1) testing VVC encoding of 1080p Standard Dynamic Range (SDR) video for various temporal resolutions i.e., frame rates from 23.98 (24/1001) fps to $120 \mathrm{fps}$.

- Test 6 requires coding sign language video in two smaller resolutions (540x960 and 360×640) which is typically shown as picture in picture. One test case covers HLG HDR (TC 6.1) and the other test case covers PQ HDR (TC 6.2) video.
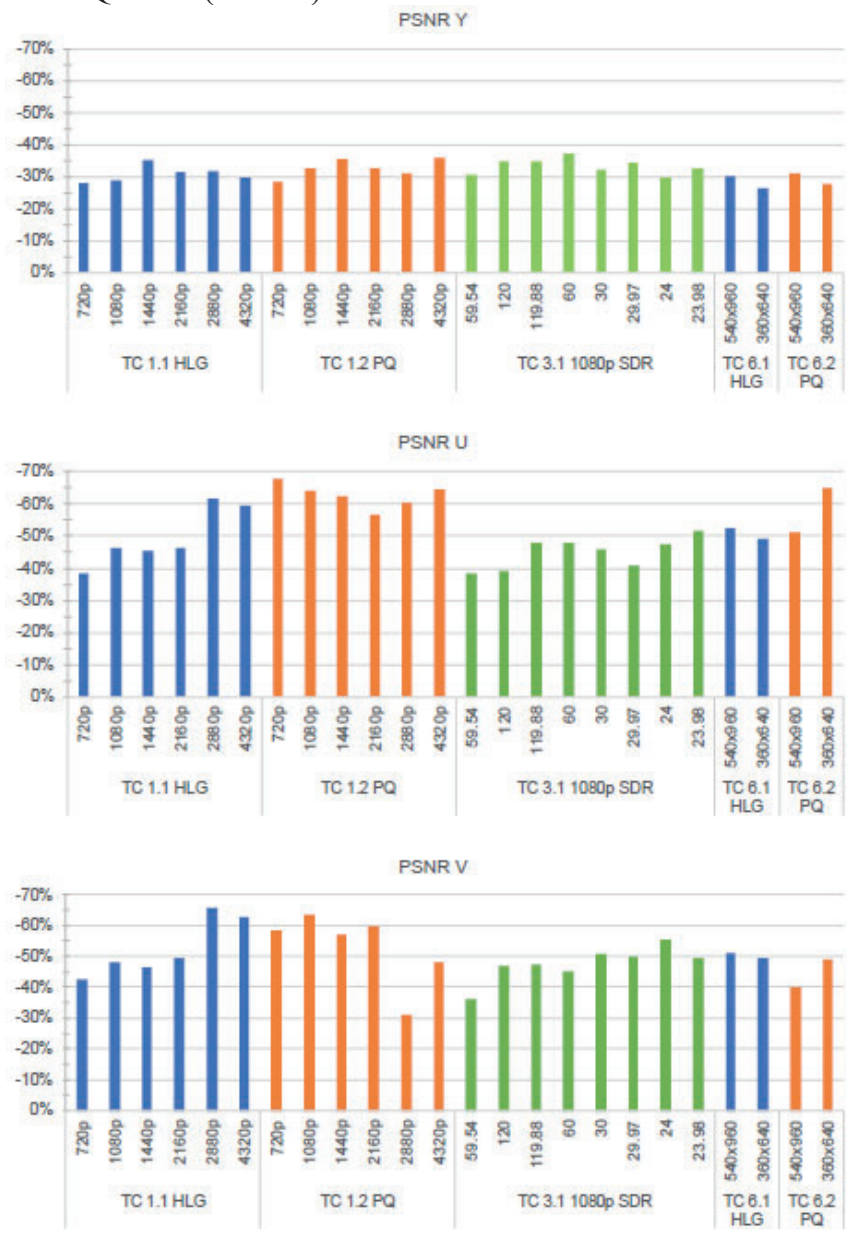

Fig. 5 : Average PSNR $Y, U$ and $V$ BD-rate savings of $V V C$ (VVenC v1.0) over HEVC (HM-16.23).

The video in all test cases uses Wide Color Gamut (WCG) colorimetry according to recommendation ITU-R BT.2100 [25]. Fig. 5 summarizes the average PSNR Bjøntegaard Delta (BD) rates for all test cases. VVC provides steady and significant bit-rate savings over HEVC for all test cases. In terms of luma PSNR Y, savings over $30 \%$ are measured. Furthermore, the chroma PSNR U and $\mathrm{V}$ savings are significantly higher, ranging between $50 \%$ and $60 \%$. This confirms that VVC has been designed for a high coding efficiency for this type of colorimetry. 


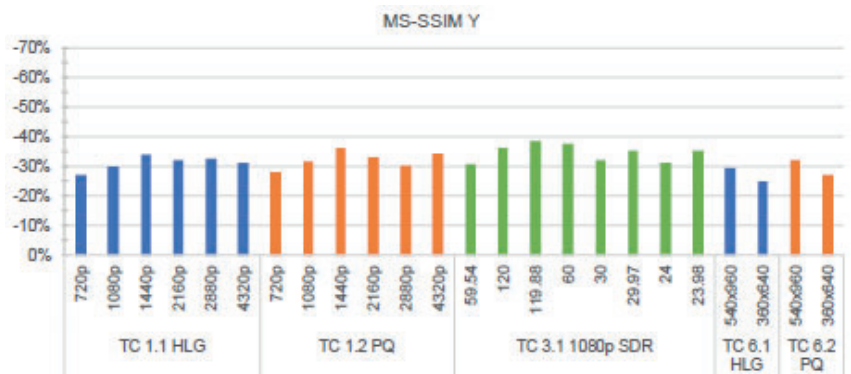

Fig. 6 : Average MS-SSIM Y BD-rate savings of VVC (VVenC v1.0) over HEVC (HM-16.23).

The average MS-SSIM Y based bit-rate savings over 30\% shown in Fig. 6 are comparable to the PSNR Y results. For the $\mathrm{U}$ and $\mathrm{V}$ component we observed a strange behavior of MS-SSIM values, especially for HDR content, which is discussed in more details in this document. For the HDR PQ content item, the weighted PSNR (wPSNR) metric has been calculated as well and is shown in Fig. 7. Compared to PSNR, the wPSNR-based BD-rate savings are slightly higher. The VVC verification tests for HD and UHD video have shown that the "real" bit-rate savings for the same perceived video quality using subjective tests and Mean Opinion Scores (MOS) tend to be higher than the ones based on objective metrics such as PSNR or MS-SSIM [26][27]. Overall, VVC is capable of coding HDR WCG video at various spatial and SDR WCG video at various temporal resolutions as well as portrait mode sign language HDR WCG and PQ WCG videos.

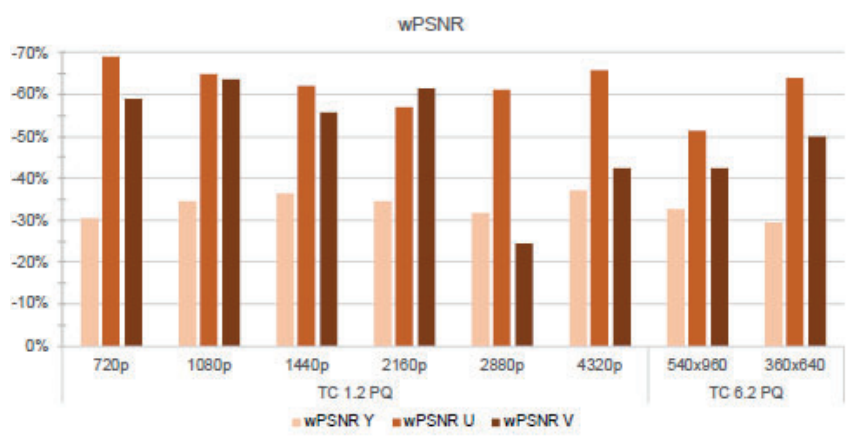

Fig. 7 : Average wPSNR BD-rate savings of VVC (VVenC v1.0) over HEVC (HM-16.23)

\section{Proposed End-To-End Transmission Chain}

\section{A. Overview}

In order to test and demonstrate VVC readiness in a live environment, we delivered to the SBTVD Forum a complete end to end transmission chain, described in the Fig. 8.

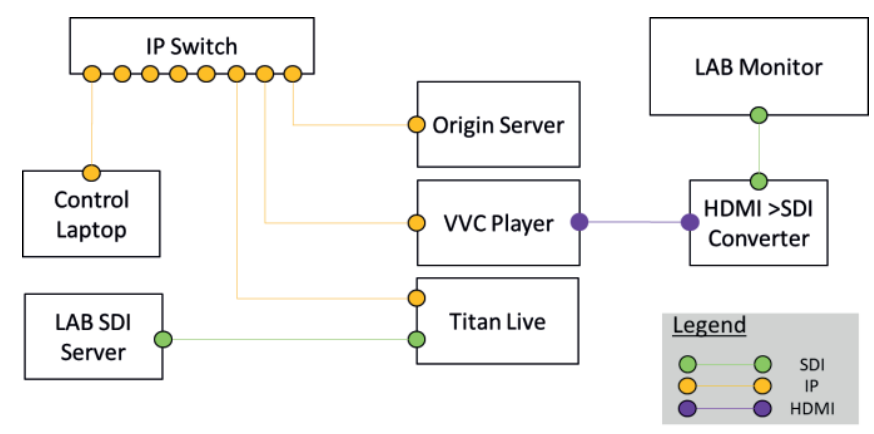

Fig. 8: Proposed end-to-end transmission scheme
The chain includes several components. A Titan Live encoder from ATEME, providing live VVC-encoding, ISOBMFF packaging and CMAF Low-Latency encapsulation. The video chunks produced are pushed to an origin server, supporting HTTP chunk transfer encoding for low-latency. The origin server is also used as host for the ad-clips to demonstrate splicing and ad-insertion use-case. A mini-PC is also provided to be used as a live VVC player, supporting live decoding as well as ad-replacement, leveraging the following open-source software [28] :

- Telecom-Paris' GPAC player [29]

- FFmpeg/FFplay [30]

- Fraunhofer HHI's VVdeC [31]

\section{B. Headend}

The ATEME TitanLive solution provides software-based implementation of a wide variety of standards for audio/video coding, packaging and transport. This solution is currently used worldwide for broadcast and OTT head-end deployments. In order to support VVC, a number of components were upgraded.

As further described in [32], VVC and HEVC present some structural similarities making an upgrade from HEVC to VVC feasible in a cost effective manner. In order to do so, the VVC syntax has been implemented with support for the tools already implemented in HEVC, disabling the other ones in the APS. Then, the HEVC tools have been upgraded to comply with VVC specification and some tools offering a good complexity-vs-gains trade-offs were implemented. Relying on the same core coding engine enabled us to leverage the existing optimized function (assembly, intrinsic) to achieve VVC real-time encoding with interesting gains over HEVC, from $10 \%$ to $15 \%$ depending on the video content. The packager has been upgraded as well to support VVC encapsulation into MPEG2-TS and ISOBMFF following FDIS specifications.

\section{Origin Server}

The ATEME origin server provided to SBTVD for TV3.0 testing has two main roles to enable end-to-end low latency. The first one is to act as an HTTP Chunked Transfer Encoding proxy able to ingest and serve data as soon as it is available. This mechanism consists of storing the HTTP chunks received from the packager in such a way that it will be able to send them to the Content Delivery Network (CDN) when requested.

The second role is to manage the requests for playlists and media segments. The origin server is responsible of blocking the request to HLS playlists if needed. When a segment and a part number are included in the request, the response will be delayed until the playlist contains at least the specified part of the segment. Regarding the request to media segments, the origin server support open range requests already described in this document. More generally and independently of the request range, the origin server will immediately serve the bytes that are already available in his cache and the send the remaining data as soon as it is received while keeping the request handler opened as long as the whole payload has not been sent. Eventually, this origin server is also hosting preencoded ad-clips used to demonstrate ad-personalization. 


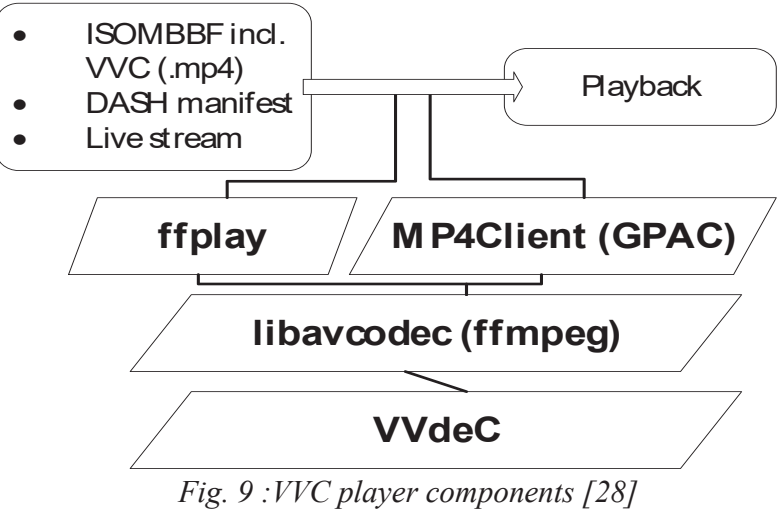

D. Player

The delivered media player relies on what's is described in [28], and illustrated in Fig. 9. It is composed of the GPAC media player and the $\mathrm{VVdeC}$ optimized VVC decoding library.

\section{1) Media player}

GPAC is a multimedia framework providing tools to package, stream and playback multimedia content. It is well-known for its MP4Box tool, an mp4 file packager and HTTP streaming (HLS) segmenter. GPAC also enables end users to build custom multimedia processing pipelines through its filterbased architecture [29]. The project is distributed under LGPL v2.1+ license.

The systems aspect of VVC have been implemented in the master branch of GPAC and are part of the nightly builds of the project. This covers the most common application use cases:

- MPEG-2 broadcasting: multiplexing and demultiplexing an MPEG2-TS program with VVC content

- MP4 file packaging and dumping of VVC bitstreams

- MPEG-DASH and HLS content packaging, with transport over HTTP or ROUTE

GPAC supports all encoders and decoders integrated in FFmpeg's libavcodec library, including VVdeC as included in [28]. The GPAC player embeds the customized libavcodec which itself embeds the VVdeC core decoding library. Until integration of VVenC is finalized in libavcodec, GPAC can consume the VVenC output through files and pipes.

\section{2) VVC decoding library}

The Fraunhofer Versatile Video Decoder, VVdeC, is an optimized VVC software decoder implementation, freely available on GitHub under a 3-clause BSD copyright license [33]. Analogue to VVenC, the license covers both commercial and non-commercial use. The latest release, v1.2.0, was published in September 2021. It is compliant with the VVC Main 10 profile and correctly decodes the current VVC conformance testing set [34]. A major part of this release is providing browser playback functionality based on open standards.

The decoder software has been derived from VTM as well, with subsequent optimizations and parallelization. The decoder allows playback of HD video at 60 frames per second (fps) on most modern computers using only 2 or 3 threads, and 60 fps UHD playback on more powerful modern workstations with sufficient processing cores to fully exploit the multi-threading potential [35].
The VVdeC package contains a simple and easy-to-use C library interface and a standalone decoder application capable of decoding elementary VVC bitstreams into raw YUV video data. The integration into the described frameworks allows actual playback of the decoded VVC content.

\section{CONCLUSION}

In this paper, the VVC proposal to the TV-3.0 CfP is described. The adequateness of VVC is discussed showing that it addresses all the TV-3.0 use-cases and requirements in an efficient manner. An overview of $\mathrm{VVC}$ is provided to highlight the key tools as well as a performance evaluation. To reinforce the relevance of VVC an end-to-end transmission chain is provided to show its readiness for commercial deployment in the context of TV-3.0 in Brazil.

\section{REFERENCES}

[1] SBTVD Forum, https://forumsbtvd.org.br/

[2] ABNT NBR 15602-1, "Digital terrestrial television - Video coding, audio coding and multiplexing, Part 1: Video Coding"

[3] T. Wiegand, G. J. Sullivan, G. Bjontegaard and A. Luthra, "Overview of the H.264/AVC video coding standard," in IEEE Transactions on Circuits and Systems for Video Technology, vol. 13, no. 7, pp. 560-576, July 2003, doi: 10.1109/TCSVT.2003.815165.

[4] B. Bross et al., "Overview of the Versatile Video Coding (VVC) Standard and its Applications, "in IEEE Transactions on Circuits and Systems for Video Technology, vol. 31, no. 10, pp. 3736-3764, Oct. 2021, doi: 10.1109/TCSVT.2021.3101953.

[5] W. Hamidouche et al., "Versatile Video Coding Standard: A Review from Coding Tools to Consumers Deployment," in IEEE Consumer Electronics Magazine, Accepted (to be published).

[6] G. J. Sullivan, J. Ohm, W. Han and T. Wiegand, "Overview of the High Efficiency Video Coding (HEVC) Standard, " in IEEE Transactions on Circuits and Systems for Video Technology, vol. 22, no. 12, pp. 1649 1668, Dec. 2012, doi: 10.1109/TCSVT.2012.2221191.

[7] F. Maurer et al., "Overview of MPEG-5 Part 2 - Low Complexity Enhancement Video Coding (LCEVC)”, in ITU Journal: ICT Discoveries, Vol. 3(1), 8 June 2020.

[8] J. Zhang, C. Jia, M. Lei, S. Wang, S. Ma and W. Gao, "Recent Development of AVS Video Coding Standard: AVS3," 2019 Picture Coding Symposium (PCS), 2019, pp. 1-5, doi: 10.1109/PCS48520.2019.8954503.

[9] SBTVD Forum, "Call for Proposals: TV 3.0 Project”, July 17, 2020 https://forumsbtvd.org.br/wp-content/uploads/2020/07/SBTVDTV-3O-CfP.pdf

[10] ATEME Press-Release, "ATEME and The Explorers to Launch the First OTT Channel Promoting VVC", https://www.ateme.com/atemeand-the-explorers-to-launch-the-first-ott-channel-promoting-vvc/

[11] ATEME Press-Release, "ATEME Joins Forces with SES to Trial FirstEver Live Over-The-Air UHD Broadcast Using VVC", https://www.ateme.com/ateme-joins-forces-with-ses-to-trial-firstever-live-over-the-air-uhd-broadcast-using-vvc

[12] ISO/IEC 23000-19:2020, "Information technology - Multimedia application format (MPEG-A) - Part 19: Common Media Application Format (CMAF) for segmented media".

[13] Information technology - Coded representation of immersive media Part 5: Visual volumetric video-based coding (V3C) and video-based point cloud compression (V-PCC), ISO/IEC 23090-5:2021

[14] SBTVD Forum, "CfP Phase 2/ Testing and Evaluation : TV3.0 Project", $\quad$ https://forumsbtvd.org.br/wpcontent/uploads/2021/03/SBTVD-TV 3 0-P2 TE 2021-03-15.pdf

[15] Huang, Yu-Wen, et al. "Block partitioning structure in the VVC standard." IEEE Transactions on Circuits and Systems for Video Technology (2021).

[16] Pfaff, Jonathan, et al. "Intra prediction and mode coding in VVC." IEEE Transactions on Circuits and Systems for Video Technology (2021).

[17] Chien, Wei-Jung, et al. "Motion Vector Coding and Block Merging in the Versatile Video Coding Standard." IEEE Transactions on Circuits and Systems for Video Technology 31.10 (2021): 3848-3861.

[18] Yang, Haitao, et al. "Subblock-based motion derivation and inter prediction refinement in versatile video coding standard." IEEE Transactions on Circuits and Systems for Video Technology (2021). 
[19] Zhao, Xin, et al. "Transform coding in the VVC standard." IEEE Transactions on Circuits and Systems for Video Technology 31.10 (2021): 3878-3890.

[20] Karczewicz, Marta, et al. "VVC in-loop filters." IEEE Transactions on Circuits and Systems for Video Technology (2021).

[21] Wang, Ye-Kui, et al. "The high-level syntax of the versatile video coding (VVC) standard." IEEE Transactions on Circuits and Systems for Video Technology (2021).

[22] Nguyen, Tung, et al. "Overview of the screen content support in VVC: Applications, coding tools, and performance." IEEE Transactions on Circuits and Systems for Video Technology (2021).

[23] A. Wieckowski et al., "VVenC: An Open And Optimized VVC Encoder Implementation," 2021 IEEE International Conference on Multimedia \& Expo Workshops (ICMEW), 2021, pp. 1-2, doi: 10.1109/ICMEW53276.2021.9455944.

[24] Fraunhofer HHI VVenC software repository. Retrieved from https://github.com/fraunhoferhhi/vvenc.

[25] ITU-R, "Image parameter values for high dynamic range television for use in production and international program exchange", Rec. ITU-R BT.2100-2, 2018

[26] V. Baroncini and M. Wien, "VVC verification test report for UHD SDR video content," doc. JVET-T2020 of ITU-T/ISO/IEC Joint Video Experts Team (JVET), 20th JVET meeting: October 2020.

[27] M. Wien and V. Baroncini, "VVC Verification Test Report for High Definition (HD) and $360^{\circ}$ Standard Dynamic Range (SDR) Video Content," doc. JVET-V2020 of ITU-T/ISO/IEC Joint Video Experts Team (JVET), 22st JVET meeting: April 2021.

[28] A. Wieckowski, C. Lehmann, B. Bross, D. Marpe, T. Biatek, M. Raulet and J. LeFeuvre, "A Complete End-To-End Open Source Toolchain for the Versatile Video Coding (VVC) Standard", In Proceedings of the ACM Multimedia Conference. ACM, October, 2021, Chengdu, CN.

[29] Jean Le Feuvre. 2020. GPAC filters. In Proceedings of the 11th ACM Multimedia Systems Conference. ACM, New York, NY, USA, 249254. DOI: https://doi.org/10.1145/3339825.3394929

[30] FFmpeg fork with full VVdeC integration. Retrieved from https://github.com/tbiat/FFmpeg/releases/tag/vvc.

[31] A. Wieckowski, G. Hege, C. Bartnik, C. Lehmann, C. Stoffers, B. Bros, and D. Marpe. 2020. Towards a Live Software Decoder Implementation for the Upcoming Versatile Video Coding (VVC) Codec. In 2020 IEEE International Conference on Image Processing (ICIP), October, 2020, Abu Dhabi, UAE 3124-3128. DOI: https://doi.org/10.1109/ICIP40778.2020.9191199

[32] Biatek, T., et al. "Future MPEG standards VVC and EVC: 8K broadcast enabler." Proc. Int. Broadcast. Conv.. 2020.

[33] Fraunhofer HHI VVdeC software repository. Retrieved from https://github.com/fraunhoferhhi/vvdec.

[34] J. Boyce, E. Alshina, F. Bossen, K. Kawamura, I. Moccagatta and W. Wan. 2021. Conformance testing for versatile video coding (Draft 6). Doc. JVET-U2008 of ITU-T/ISO/IEC Joint Video Experts Team (JVET), 21st JVET meeting: January 2021.

[35] A. Wieckowski, G. Hege, C. Bartnik, C. Lehmann, C. Stoffers, B. Bros, and D. Marpe. 2020. Towards a Live Software Decoder Implementation for the Upcoming Versatile Video Coding (VVC) Codec. In 2020 IEEE International Conference on Image Processing (ICIP), October, 2020, Abu Dhabi, UAE 3124-3128. DOI: https://doi.org/10.1109/ICIP40778.2020.9191199

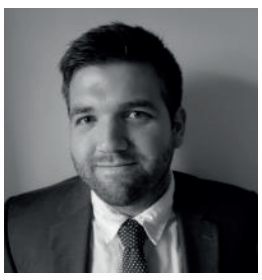

Thibaud Biatek received the Ph.D. degree in signal and image processing from the Institut National des Sciences Appliquées, Rennes, France, in 2016. From 2013 to 2017, he was a Doctoral and Post-Doctoral Fellow with TDF, Cesson-Sévigné, France. From 2017 to 2019, he was a Video Coding Expert with TDF, where he was involved in MPEG and DVB standardization activities. In 2019, he was a Senior Engineer with Qualcomm working on VVC standardization. Since 2020, he has been Director of Technology and Standards with ATEME, working on partnership projects and multimedia standards, contributing to MPEG, DVB and 3GPP groups. His research interests include compression, processing, and delivery of audiovisual signals over broadcast and broadband networks.

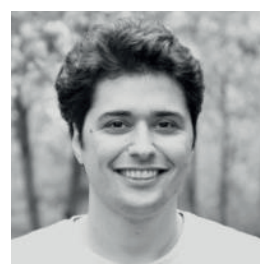

Mohsen Abdoli received his Master of Engineering degree in 2013 from Sharif University of Technology, Tehran, Iran, and his Doctor of Philosophy in 2018, jointly from Université Paris-Saclay and CentraleSupélec, Paris, France. In 2015, he joined Orange Labs, Rennes, France, where he actively contributed to development of several video compression techniques, targeting the VVC standard by JVET, particularly in the domain of intra prediction and residual coding of natural/screen content. In 2018, he joined Ateme, Rennes, France, where he contributed to the worldfirst implementation of real-time VVC transcoder for VoD and live streaming applications. He is currently with IRT $\mathrm{b}<>$ com, Rennes, France, holding the position of standardization engineer. His research interests include areas of signal processing, encoder optimization, machine learning and quality assessment.

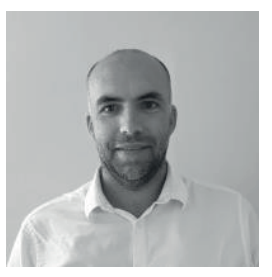

Mickaël Raulet is CTO at ATEME, where he drives research and innovation with various collaborative $R \& D$ projects. He represents ATEME in several standardization bodies: ATSC, DVB，3GPP，ISO/IEC，ITU，MPEG, DASH-IF, CMAF-IF, SVA and UHDForum. He is the author of numerous patents and more than 100 conference and journal scientific papers. In 2006 he received his Ph.D. from INSA in electronic and signal processing, in collaboration with Mitsubishi Electric ITE (Rennes, France).

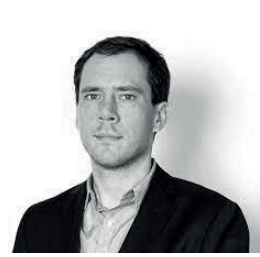

Adam Wieckowski received the M.Sc. degree in computer engineering from the Technical University of Berlin, Berlin, Germany, in 2014.,In 2016, he joined the Fraunhofer Institute for Telecommunications-Heinrich Hertz Institute, Berlin, as a Research Assistant. He worked on the development of the software, which later became the test model for VVC Development. He contributed several technical contributions during the standardization of VVC. Since 2019, he has been a Project Manager coordinating the technical development of decoder and encoder solutions for the VVC standard.

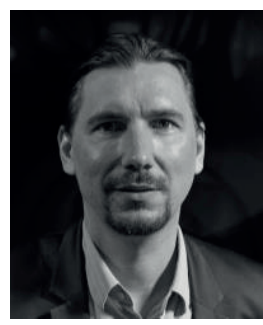

Christian Lehmann received the M.S. degree in computer science from University Leipzig, Germany, in 2006. He joined the Fraunhofer Institute for Telecom-munications - Heinrich Hertz Institute, Berlin, Germany in 2009, where he is a research associate in the Video Coding Systems group.His research interests include the development of optimized software video codecs, multimedia frameworks and systems integration. This includes real-time video decoder and encoder solutions for High Efficiency Video Coding (HEVC) and the Versatile Video Coding (VVC) open source implementations VVenC and VVdeC. 


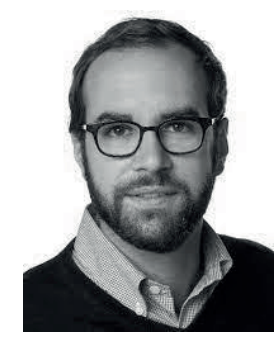

Benjamin Bross received the Dipl.-Ing. degree in electrical engineering from RWTH Aachen University, Aachen, Germany, in 2008. In 2009, he joined the Fraunhofer Institute for Telecommunications - Heinrich Hertz Institute, Berlin, Germany, where he is currently heading the Video Coding Systems group at the Video Coding \& Applications Department and in 2011, he became a part-time lecturer at the HTW University of Applied Sciences Berlin. Since 2010, Benjamin is very actively involved in the ITU-T VCEG | ISO/IEC MPEG video coding standardization processes as a technical contributor, coordinator of core experiments and chief editor of the High Efficiency Video Coding (HEVC) standard [ITU-T H.265 | ISO/IEC 23008-2] and the new Versatile Video Coding (VVC) standard [ITU-T H.266 | ISO/IEC 23090-3]. In addition to his involvement in standardization, his group is developing standard-compliant software implementations. This includes the development of an HEVC live software encoder that is currently deployed in broadcast for HD and UHD TV channels and most recently, the open and optimized VVC software implementations VVenC and VVdeC. Benjamin Bross is an author or coauthor of several fundamental HEVC and VVC-related publications, and an author of two book chapters on HEVC and Inter-Picture Prediction Techniques in HEVC. He received the IEEE Best Paper Award at the 2013 IEEE International Conference on Consumer Electronics - Berlin in 2013, the SMPTE Journal Certificate of Merit in 2014 and an Emmy Award at the 69th Engineering Emmy Awards in 2017 as part of the Joint Collaborative Team on Video Coding for its development of HEVC.

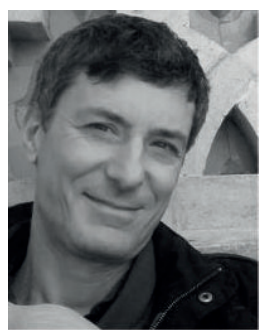

Edouard François is currently Principal Scientist at InterDigital where he is leading a research team focused on video and media compression. In previous years, he has been part of Technicolor and Canon research. He received an Engineering degree from the IMT Atlantique, France and a Ph.D. degree in computer science from University of Rennes 1, France. He has been participating to several standardization activities, including the specifications of the scalable extension of AVC/H.264 (known as SVC), the HEVC/H.265 standard and its extensions, and the recent VVC/H.266 standard. He had previously co-chaired the MPEG activity on HDR and WCG video coding. His main research interests include signal and video processing, conventional and ML-based video coding, and high dynamic range video.

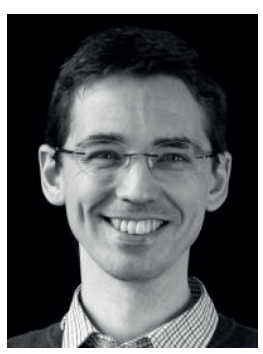

Philippe de Lagrange received the Eng. degree in electrical engineering from the INSA of Rennes, France, in 1999. He is currently with Interdigital R\&D France, in the Core Video Coding team, where he has contributed to VVC through his active participation to the JVET group. He also leads a team that promotes VVC through optimized software implementations (e.g. full-featured multi-threaded VTM decoder, including multi-layer), performance testing, publications, partnerships, and participation to applicative standards. He provided the system infrastructure for offline testing of VVC and remote support during the evaluation phase of TV3.0 in Brazil. Previously, he worked on broadcast video encoders (TVN/Harmonic and Envivio/MediaKind), decoders (Technicolor), and also worked for a radiocommunication research lab (Mitsubishi ITE), and in satellite positioning R\&D (Galileo).

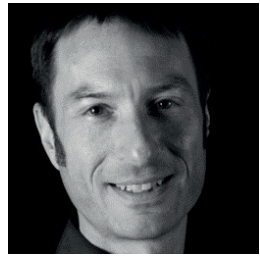

Ralf Schaefer received his engineering degree (Dipl.-Ing.) from the Technical University Kaiserslautern/Germany and joined InterDigital in June 2019. As full time standard professional, Ralf chairs the MPEG WG7 AhG on Video-based Graphics Coding, is vice-chair of the DVB Commercial Module and is elected member of the DVB Steering Board. Furthermore, Ralf is an active contributor to standards working groups in DVB, ETSI, SBTVD Brazil, NorDiG and FAVN France. Previously he chaired the MPEG 3DG AhG on Point Cloud Compression, which advanced the work on Video-based Point Cloud Compression (V-PCC) between MPEG meetings and led to the publication of ISO/IEC 23090-5:2021. Before joining Interdigital, Ralf occupied a similar position in Technicolor, where he contributed to ATSC3.0 and NGBF South Korea. Earlier he held R\&D positions at various levels in Thomson and chaired working groups in DVB related to IPTV, Home Networking and Companion Screens.

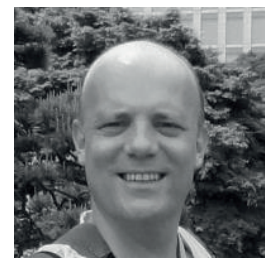

Jean Le Feuvre received his Ingénieur (M.Sc.) degree in Telecommunications in 1999, from TELECOM Bretagne. He has been involved in MPEG standardization since 2000 for his NYCbased startup Avipix,llc and joined TELECOM Paris in 2005 as Research Engineer within the Image, Data and Signal Department. His main research topics cover multimedia authoring, delivery and rendering systems in broadcast, broadband and home networking environments. $\mathrm{He}$ is the project leader and maintainer of GPAC, a multimedia framework based on standard technologies (MPEG, W3C, IETF). He is the author of many scientific contributions (peer-reviewed journal articles, conference papers, book chapters, patents) in the field and is editor of several ISO standards." 